\title{
Common neighborhood spectrum of commuting graphs of finite groups
}

\author{
W. N. T. Fasfous, R. Sharafdini, and R. K. Nath*
}

\author{
Communicated by I. Ya. Subbotin
}

\begin{abstract}
A BSTRACT. The commuting graph of a finite non-abelian group $G$ with center $Z(G)$, denoted by $\Gamma_{c}(G)$, is a simple undirected graph whose vertex set is $G \backslash Z(G)$, and two distinct vertices $x$ and $y$ are adjacent if and only if $x y=y x$. In this paper, we compute the common neighborhood spectrum of commuting graphs of several classes of finite non-abelian groups and conclude that these graphs are $\mathrm{CN}$-integral.
\end{abstract}

\section{Introduction}

Let $\mathcal{G}$ be a simple graph whose vertex set is $V(\mathcal{G})=\left\{v_{1}, v_{2}, \ldots, v_{n}\right\}$. The common neighborhood of two distinct vertices $v_{i}$ and $v_{j}$, denoted by $C\left(v_{i}, v_{j}\right)$, is the set of vertices adjacent to both $v_{i}$ and $v_{j}$ other than $v_{i}$ and $v_{j}$. The common neighborhood matrix of $\mathcal{G}$, denoted by $C N(\mathcal{G})$, is a matrix of size $n$ whose $(i, j)$ th entry is 0 or $\left|C\left(v_{i}, v_{j}\right)\right|$ according as $i=j$ or $i \neq j$. Alwardi et al. have introduced and studied this matrix in [4]. The set of all the eigenvalues of $C N(\mathcal{G})$ with multiplicities denoted by $\mathrm{CN}-\operatorname{spec}(\mathcal{G})$ is called the common neighborhood spectrum, in short CN-spectrum, of $\mathcal{G}$. If $\alpha_{1}, \alpha_{2}, \ldots, \alpha_{k}$ are the eigenvalues of $C N(\mathcal{G})$ with multiplicities $a_{1}, a_{2}, \ldots, a_{k}$ respectively then we write $\mathrm{CN}-\operatorname{spec}(\mathcal{G})=$ $\left\{\alpha_{1}^{a_{1}}, \alpha_{2}^{a_{2}}, \ldots, \alpha_{k}^{a_{k}}\right\}$. A graph $\mathcal{G}$ is called CN-integral if $\mathrm{CN}-\operatorname{spec}(\mathcal{G})$ contains only integers.

${ }^{*}$ Corresponding author.

2020 MSC: 20D99, 05C50, 15A18, 05C25.

Key words and phrases: commuting graph, spectrum, integral graph, finite group. 
The commuting graph of a finite non-abelian group $G$ with center $Z(G)$ is a simple undirected graph whose vertex set is $G \backslash Z(G)$ and two vertices $x$ and $y$ are adjacent if and only if $x y=y x$. We write $\Gamma_{c}(G)$ to denote this graph. In $[5,12-14,16,18,21,23]$, various aspects of $\Gamma_{c}(G)$ are studied. In section 2 of this paper, we derive a computing formula for CN-spectrum of a particular class of graphs and list a few useful results. In section 3, we compute CN-spectrum of commuting graph of groups $G$ such that $\frac{G}{Z(G)}$ is isomorphic to the Suzuki group of order $20, \mathbb{Z}_{p} \times \mathbb{Z}_{p}$ (where $p$ is a prime) and a dihedral group of order $2 m$. In section 4 , we compute CN-spectrum of commuting graphs of several well-known groups including the quasidihedral groups, projective special linear groups, general linear groups etc. As consequences of our results, in section 5, we show that commuting graphs of all the groups considered in section 3 and section 4 are CN-integral. We shall determine some positive integers $n$ such that $\Gamma_{c}(G)$ is CN-integral if $G$ is an $n$-centralizer group. Recall that a group $G$ is called an $n$-centralizer group if $|\operatorname{Cent}(G)|=n$, where $\operatorname{Cent}(G)=\left\{C_{G}(x): x \in G\right\}$ and $C_{G}(x)=\{y \in G: x y=y x\}$ is the centralizer of $x$. The study of $n$-centralizer groups was initiated by Belcastro and Sherman [7] in 1994. The reader may conf. [11] for various results on $n$-centralizer groups. We shall also determine some positive rational numbers $r$ such that $\Gamma_{c}(G)$ is CN-integral if the commutativity degree of $G$ is $r$. Recall that the commutativity degree of $G$, denoted by $\operatorname{Pr}(G)$, is the probability that a randomly chosen pair of elements of $G$ commute. The origin of commutativity degree of a finite group lies in a paper of Erdös and Turán (see [15]). The reader may conf. [8, 9, 19, 22] for various results regarding this notion. Further, we show that $\Gamma_{c}(G)$ is CN-integral if $\Gamma_{c}(G)$ is planar or toroidal and $G$ is not isomorphic to $S_{4}$, the symmetric group of degree 4 . Note that a graph is planar or toroidal according as its genus is zero or one respectively. Also, the genus of a graph is the smallest non-negative integer $n$ such that the graph can be embedded on the surface obtained by attaching $n$ handles to a sphere. It is worth mentioning that Afkhami et al. [3] and Das et al. [10] have classified all finite non-abelian groups whose commuting graphs are planar or toroidal recently.

\section{A useful formula and prerequisites}

We write $\mathcal{G}=\mathcal{G}_{1} \sqcup \mathcal{G}_{2}$ to denote that $\mathcal{G}$ has two components namely $\mathcal{G}_{1}$ and $\mathcal{G}_{2}$. Also, $l K_{m}$ denotes the disjoint union of $l$ copies of the complete graph $K_{m}$ on $m$ vertices. We begin this section with the following lemma. 
Lemma 1. If $\mathcal{G}=\mathcal{G}_{1} \sqcup \mathcal{G}_{2} \sqcup \cdots \sqcup \mathcal{G}_{m}$ then $\operatorname{CN}-\operatorname{spec}(\mathcal{G})=\bigcup_{i=1}^{k} \operatorname{CN}-\operatorname{spec}\left(\mathcal{G}_{i}\right)$ counting the multiplicities.

Lemma 2. If $K_{n}$ denotes the complete graph on $n$ vertices then

$$
\mathrm{CN}-\operatorname{spec}\left(K_{n}\right)=\left\{(-(n-2))^{n-1},((n-1)(n-2))^{1}\right\} .
$$

Proof. Let $A\left(K_{n}\right)$ be the adjacency matrix of $K_{n}$. Then we have $C N\left(K_{n}\right)$ $=(n-2) A\left(K_{n}\right)$. Hence, the result follows.

Now we derive a formula for CN-spectrum of graphs that are disjoint union of some complete graphs. The following theorem is very useful in order to compute CN-spectrum of commuting graphs of some classes of finite groups.

Theorem 1. Let $\mathcal{G}=l_{1} K_{m_{1}} \sqcup l_{2} K_{m_{2}} \sqcup \cdots \sqcup l_{k} K_{m_{k}}$, where $l_{i} K_{m_{i}}$ denotes disjoint union of $l_{i}$ copies of the complete graphs $K_{m_{i}}$ on $m_{i}$ vertices for $1 \leqslant i \leqslant k$. Then

$$
\begin{aligned}
\mathrm{CN}-\operatorname{spec}(\mathcal{G})=\left\{\left(-\left(m_{1}-2\right)\right)^{l_{1}\left(m_{1}-1\right)},\left(\left(m_{1}-1\right)\left(m_{1}-2\right)\right)^{l_{1}}, \ldots,\right. \\
\left.\left(-\left(m_{k}-2\right)\right)^{l_{k}\left(m_{k}-1\right)},\left(\left(m_{k}-1\right)\left(m_{k}-2\right)\right)^{l_{k}}\right\} .
\end{aligned}
$$

Proof. Let $\mathcal{G}=\mathcal{G}_{1} \sqcup \mathcal{G}_{2} \sqcup \cdots \sqcup \mathcal{G}_{k}$. Then, by Lemma 1, we have $\operatorname{CN}-\operatorname{spec}(\mathcal{G})$ $=\cup_{i=1}^{k} \mathrm{CN}-\operatorname{spec}\left(\mathcal{G}_{i}\right)$ counting the multiplicities. Therefore, using Lemma 2, we have

$$
\text { CN-spec }\left(l_{i} K_{m_{i}}\right)=\left\{\left(-\left(m_{i}-2\right)\right)^{l_{i}\left(m_{i}-1\right)},\left(\left(m_{i}-1\right)\left(m_{i}-2\right)\right)^{l_{i}}\right\} .
$$

Hence, the result follows by considering $\mathcal{G}_{i}=l_{i} K_{m_{i}}$ for $1 \leqslant i \leqslant k$.

We conclude this section with the following useful results.

Theorem 2. [7, Theorem 2] If $G$ is a finite 4-centralizer group then $\frac{G}{Z(G)} \cong \mathbb{Z}_{2} \times \mathbb{Z}_{2}$

Theorem 3. [6, Lemma 2.7] If $G$ is a finite $(p+2)$-centralizer $p$-group then $\frac{G}{Z(G)} \cong \mathbb{Z}_{p} \times \mathbb{Z}_{p}$.

Theorem 4. [7, Theorem 4] If $G$ is a finite 5-centralizer group then $\frac{G}{Z(G)} \cong \mathbb{Z}_{3} \times \mathbb{Z}_{3}$ or $D_{6}$. 
Theorem 5. [2, Lemma 2.4] Let $G$ be a finite non-abelian group and $\left\{x_{1}, x_{2}, \ldots, x_{r}\right\}$ be a set of pairwise non-commuting elements of $G$ having maximal size. Then $G$ is a 4 -centralizer or a 5 -centralizer group according as $r=3$ or 4 .

Theorem 6. [17, Theorem 3] Let $G$ be a finite group and $p$ the smallest prime divisor of $|G|$. Then $\operatorname{Pr}(G)=\frac{p^{2}+p-1}{p^{3}}$ if and only if $\frac{G}{Z(G)} \cong \mathbb{Z}_{p} \times \mathbb{Z}_{p}$.

Theorem 7. [8, Proposition 3.3.7] If $G$ is a finite non-solvable group with $\operatorname{Pr}(G)=\frac{1}{12}$ then $G \cong A_{5} \times B$ for some finite abelian group $B$.

Theorem 8. [3, Theorem 2.2] Let $G$ be a finite non-abelian group. Then $\Gamma_{c}(G)$ is planar if and only if $G$ is isomorphic to either $D_{6}, D_{8}, D_{10}$, $D_{12}, Q_{8}, Q_{12}, \mathbb{Z}_{2} \times D_{8}, \mathbb{Z}_{2} \times Q_{8}, \mathcal{M}_{16}, \mathbb{Z}_{4} \rtimes \mathbb{Z}_{4}, D_{8} * \mathbb{Z}_{4}, S G(16,3), A_{4}, A_{5}, S_{4}$, $S L(2,3)$ or $S z(2)$.

Theorem 9. [10, Theorem 6.6] Let $G$ be a finite non-abelian group. Then $\Gamma_{c}(G)$ is toroidal if and only if $G$ is isomorphic to either $D_{14}, D_{16}$, $Q_{16}, Q D_{16}, D_{6} \times \mathbb{Z}_{3}, A_{4} \times \mathbb{Z}_{2}$ or $\mathbb{Z}_{7} \rtimes \mathbb{Z}_{3}$.

Theorem 10. [1, Proposition 2. 3] Let $G$ be a finite non-abelian group. Then the complement of $\Gamma_{c}(G)$ is planar if and only if $G$ is isomorphic to either $D_{6}, D_{8}$ or $Q_{8}$.

\section{Groups having known central quotient}

In this section, we compute CN-spectrum of commuting graphs of finite non-abelian groups having well-known central quotient such as the Suzuki group of order $20, \mathbb{Z}_{p} \times \mathbb{Z}_{p}$ (where $p$ is a prime) and the dihedral groups. We begin with the following lemma from [12] and [13].

Lemma 3. Let $G$ be a finite group with center $Z(G)$. If $\frac{G}{Z(G)}$ is isomorphic to

(a) the Suzuki group $S z(2)$, presented by $\left\langle a, b: a^{5}=b^{4}=1, b^{-1} a b=a^{2}\right\rangle$, then $\Gamma_{c}(G)=K_{4|Z(G)|} \sqcup 5 K_{3|Z(G)|}$.

(b) $\mathbb{Z}_{p} \times \mathbb{Z}_{p}$, where $p$ is a prime, then $\Gamma_{c}(G)=(p+1) K_{(p-1)|Z(G)|}$.

(c) the dihedral group $D_{2 m}(m \geqslant 2)$, presented by $\left\langle a, b: a^{m}=b^{2}=1\right.$, $\left.b a b^{-1}=a^{-1}\right\rangle$, then $\Gamma_{c}(G)=K_{(m-1)|Z(G)|} \sqcup m K_{|Z(G)|}$.

Now we have the following main result of this section.

Theorem 11. Let $G$ be a finite group with center $Z(G)$. If $\frac{G}{Z(G)}$ is isomorphic to 
(a) the Suzuki group $S z(2)$, presented by $\left\langle a, b: a^{5}=b^{4}=1, b^{-1} a b=a^{2}\right\rangle$, then $\mathrm{CN}-\operatorname{spec}\left(\Gamma_{c}(G)\right)$ is given by

$$
\begin{aligned}
& \left\{(-(4 \mid Z(G)-2))^{4 \mid Z(G)-1},((4 \mid Z(G)-1)(4 \mid Z(G)-2))^{1},\right. \\
& \left.\quad(-(3|Z(G)|-2))^{5(3|Z(G)|-1)},((3|Z(G)|-1)(3|Z(G)|-2))^{5}\right\} .
\end{aligned}
$$

(b) $\mathbb{Z}_{p} \times \mathbb{Z}_{p}$, where $p$ is a prime, then $\mathrm{CN}-\operatorname{spec}\left(\Gamma_{c}(G)\right)$ is given by

$$
\begin{aligned}
\left\{(-((p-1)|Z(G)|-2))^{(p+1)((p-1)|Z(G)|-1)}\right. & \\
& \left.(((p-1)|Z(G)|-1)((p-1)|Z(G)|-2))^{p+1}\right\} .
\end{aligned}
$$

(c) the dihedral group $D_{2 m}(m \geqslant 2)$, presented by $\left\langle a, b: a^{m}=b^{2}=\right.$ $\left.1, b a b^{-1}=a^{-1}\right\rangle$, then $\mathrm{CN}-\operatorname{spec}\left(\Gamma_{c}(G)\right)$ is given by

$$
\begin{aligned}
& \left\{(-((m-1)|Z(G)|-2))^{(m-1)|Z(G)|-1},\right. \\
& \quad(((m-1)|Z(G)|-1)((m-1)|Z(G)|-2))^{1}, \\
& \left.\quad(-(|Z(G)|-2))^{m(|Z(G)|-1)},((|Z(G)|-1)(|Z(G)|-2))^{m}\right\} .
\end{aligned}
$$

Proof. (a) If $\frac{G}{Z(G)} \cong S z(2)$ then, by Lemma $3\left(\right.$ a), we have $\Gamma_{c}(G)=$ $K_{4|Z(G)|} \sqcup 5 K_{3|Z(G)|}$. Therefore, by Theorem 1, we have

$\mathrm{CN}-\operatorname{spec}\left(\Gamma_{c}(G)\right)=$

$$
\begin{aligned}
& \left\{(-(4 \mid Z(G)-2))^{4 \mid Z(G)-1},((4 \mid Z(G)-1)(4 \mid Z(G)-2))^{1},\right. \\
& \left.\quad(-(3|Z(G)|-2))^{5(3|Z(G)|-1)},((3|Z(G)|-1)(3|Z(G)|-2))^{5}\right\} .
\end{aligned}
$$

(b) If $\frac{G}{Z(G)} \cong \mathbb{Z}_{p} \times \mathbb{Z}_{p}$ then, by Lemma $3(\mathrm{~b})$, we have $\Gamma_{c}(G)=$ $(p+1) K_{(p-1)|Z(G)|}$. Therefore, by Theorem 1, we have

$$
\begin{aligned}
\mathrm{CN}-\operatorname{spec}\left(\Gamma_{c}(G)\right)=\left\{(-((p-1)|Z(G)|-2))^{(p+1)((p-1)|Z(G)|-1)},\right. \\
\left.(((p-1)|Z(G)|-1)((p-1)|Z(G)|-2))^{p+1}\right\} .
\end{aligned}
$$

(c) If $\frac{G}{Z(G)} \cong D_{2 m}$ then, by Lemma 3(c), we have

$$
\Gamma_{c}(G)=K_{(m-1)|Z(G)|} \sqcup m K_{|Z(G)|} \cdot
$$

Therefore, by Theorem 1,

$$
\begin{aligned}
& \mathrm{CN}-\operatorname{spec}(\left.\Gamma_{c}(G)\right)=\left\{(-((m-1)|Z(G)|-2))^{(m-1)|Z(G)|-1},\right. \\
&(((m-1)|Z(G)|-1)((m-1)|Z(G)|-2))^{1}, \\
&\left.\quad(-(|Z(G)|-2))^{m(|Z(G)|-1)},((|Z(G)|-1)(|Z(G)|-2))^{m}\right\} .
\end{aligned}
$$

This completes the proof. 
We conclude this section with the following corollaries of Theorem 11.

Corollary 1. Let $G$ be a group isomorphic to one of the following groups

(a) $\mathbb{Z}_{2} \times D_{8}$

(b) $\mathbb{Z}_{2} \times Q_{8}$

(c) $\mathcal{M}_{16}=\left\langle a, b: a^{8}=b^{2}=1, b a b=a^{5}\right\rangle$

(d) $\mathbb{Z}_{4} \rtimes \mathbb{Z}_{4}=\left\langle a, b: a^{4}=b^{4}=1, b a b^{-1}=a^{-1}\right\rangle$

(e) $D_{8} * \mathbb{Z}_{4}=\left\langle a, b, c: a^{4}=b^{2}=c^{2}=1, a b=b a, a c=c a, b c=a^{2} c b\right\rangle$

(f) $S G(16,3)=\left\langle a, b: a^{4}=b^{4}=1, a b=b^{-1} a^{-1}, a b^{-1}=b a^{-1}\right\rangle$.

Then CN-spec $\left(\Gamma_{c}(G)\right)=\left\{(-2)^{9}, 6^{3}\right\}$.

Proof. We have $|G|=16$ and $|Z(G)|=4$. Therefore, $\frac{G}{Z(G)} \cong \mathbb{Z}_{2} \times \mathbb{Z}_{2}$. Hence, putting $p=2$ and $|Z(G)|=4$ in Theorem $11(\mathrm{~b})$ we get the required result.

Corollary 2. Let $G$ be a non-abelian group.

(a) If $G$ is of order $p^{3}$, for any prime $p$, then

$$
\begin{aligned}
\mathrm{CN}-\operatorname{spec}\left(\Gamma_{c}(G)\right)=\left\{\left(-\left(p^{2}-p-2\right)\right)^{(p+1)\left(p^{2}-p-1\right)}\right. \\
\\
\left.\left(\left(p^{2}-p-1\right)\left(p^{2}-p-2\right)\right)^{p+1}\right\} .
\end{aligned}
$$

(b) Let $G$ be the metacyclic group $M_{2 m n}(m \geqslant 3)$, presented by $\langle a, b$ : $\left.a^{m}=b^{2 n}=1, b a b^{-1}=a^{-1}\right\rangle$. If $m$ is odd then $\mathrm{CN}-\operatorname{spec}\left(\Gamma_{c}\left(M_{2 m n}\right)\right)$ is given by

$$
\begin{aligned}
&\left\{(-(m n-n-2))^{m n-n-1},\right.( \\
&(m n-n-1)(m n-n-2))^{1}, \\
&\left.(-(n-2))^{m n-m},((n-1)(n-2))^{m}\right\} .
\end{aligned}
$$

If $m$ is even then $\mathrm{CN}-\operatorname{spec}\left(\Gamma_{c}\left(M_{2 m n}\right)\right)$ is given by

$$
\begin{aligned}
& \left\{(-(m n-2 n-2))^{m n-2 n-1},((m n-2 n-1)(m n-2 n-2))^{1},\right. \\
& \left.(-(2 n-2))^{\frac{m(2 n-1)}{2}},((2 n-1)(2 n-2))^{\frac{m}{2}}\right\} .
\end{aligned}
$$

(c) If $G$ is the dihedral group $D_{2 m}(m \geqslant 3)$, presented by $\left\langle a, b: a^{m}=\right.$ $\left.b^{2}=1, b a b^{-1}=a^{-1}\right\rangle$, then

$$
\begin{aligned}
& \text { CN-spec }\left(\Gamma_{c}(G)\right) \\
& = \begin{cases}\left\{(-(m-3))^{m-2},((m-2)(m-3))^{1}, 0^{m}\right\}, & \text { if } m \text { is odd } \\
\left\{(-(m-4))^{m-3},((m-3)(m-4))^{1}, 0^{m}\right\}, & \text { if } m \text { is even. }\end{cases}
\end{aligned}
$$

(d) If $G$ is the generalized quaternion group $Q_{4 n}(n \geqslant 2)$, presented by $\left\langle x, y: y^{2 n}=1, x^{2}=y^{n}, x y x^{-1}=y^{-1}\right\rangle$, then

$$
\mathrm{CN}-\operatorname{spec}\left(\Gamma_{c}(G)\right)=\left\{(-(2 n-4))^{2 n-3},((2 n-3)(2 n-4))^{1}, 0^{2 n}\right\} .
$$


Proof. (a) If $G$ is of order $p^{3}$ then $|Z(G)|=p$ and $\frac{G}{Z(G)} \cong \mathbb{Z}_{p} \times \mathbb{Z}_{p}$. Therefore, putting $|Z(G)|=p$, in Theorem 11(b), we get

$\mathrm{CN}-\operatorname{spec}\left(\Gamma_{c}(G)\right)=$

$$
\left\{\left(-\left(p^{2}-p-2\right)\right)^{(p+1)\left(p^{2}-p-1\right)},\left(\left(p^{2}-p-1\right)\left(p^{2}-p-2\right)\right)^{p+1}\right\} .
$$

(b) If $m$ is odd then $\left|Z\left(M_{2 m n}\right)\right|=n$ and $\frac{M_{2 m n}}{Z\left(M_{2 m n}\right)} \cong D_{2 m}$. Therefore, putting $|Z(G)|=n$, in Theorem 11(c), we get

$$
\begin{aligned}
& \mathrm{CN}-\operatorname{spec}\left(\Gamma_{c}\left(M_{2 m n}\right)\right)=\left\{(-(m n-n-2))^{m n-n-1},\right. \\
& \left.\quad((m n-n-1)(m n-n-2))^{1},(-(n-2))^{m n-m},((n-1)(n-2))^{m}\right\} .
\end{aligned}
$$

If $m$ is even then $\left|Z\left(M_{2 m n}\right)\right|=2 n$ and $\frac{M_{2 m n}}{Z\left(M_{2 m n}\right)} \cong D_{m}$. Therefore, putting $|Z(G)|=2 n$ and replacing $m$ by $\frac{m}{2}$, in Theorem 11(c), we get

$\mathrm{CN}-\operatorname{spec}\left(\Gamma_{c}\left(M_{2 m n}\right)\right)=$

$$
\begin{array}{r}
\left\{(-(m n-2 n-2))^{m n-2 n-1},((m n-2 n-1)(m n-2 n-2))^{1},\right. \\
\left.(-(2 n-2))^{\frac{m(2 n-1)}{2}},((2 n-1)(2 n-2))^{\frac{m}{2}}\right\} .
\end{array}
$$

(c) Follows from part (b), considering $n=1$.

(d) Note that $\left|Z\left(Q_{4 n}\right)\right|=2$ and $\frac{Q_{4 n}}{Z\left(Q_{4 n}\right)} \cong D_{2 n}$. Therefore, putting $|Z(G)|=2$ and $m=n$ in Theorem 11(c), we get the required result.

\section{More classes of groups}

In this section, we compute CN-spectrum of commuting graphs of several well-known groups including the quasidihedral groups, projective special linear groups, general linear groups etc. We begin with the following useful results from [12].

Lemma 4. Let $G$ be a non-abelian group. If $G$ is isomorphic to

(a) a group of order $p q$, where $p$ and $q$ are primes with $p \mid(q-1)$, then $\Gamma_{c}(G)=K_{q-1} \sqcup q K_{p-1}$.

(b) the quasidihedral group $Q D_{2^{n}}(n \geqslant 4)$, presented by $\left\langle a, b: a^{2^{n-1}}=\right.$ $\left.b^{2}=1, b a b^{-1}=a^{2^{n-2}-1}\right\rangle$, then $\Gamma_{c}(G)=K_{2^{n-1}-2} \sqcup 2^{n-2} K_{2}$.

(c) the projective special linear group $\operatorname{PSL}\left(2,2^{k}\right)$, where $k \geqslant 2$, then $\Gamma_{c}(G)=\left(2^{k}+1\right) K_{2^{k}-1} \sqcup 2^{k-1}\left(2^{k}+1\right) K_{2^{k}-2} \sqcup 2^{k-1}\left(2^{k}-1\right) K_{2^{k}}$.

(d) the general linear group $G L(2, q)$, where $q=p^{n}>2$ and $p$ is a prime, then

$$
\Gamma_{c}(G)=\frac{q(q+1)}{2} K_{q^{2}-3 q+2} \sqcup \frac{q(q-1)}{2} K_{q^{2}-q} \sqcup(q+1) K_{q^{2}-2 q+1} .
$$


Lemma 5. Let $G$ be a non-abelian group. If $G$ is isomorphic to

(a) the Hanaki group $A(n, \vartheta)(n \geqslant 2)$ of order $2^{2 n}$ given by

$$
\left\{U(a, b)=\left[\begin{array}{ccc}
1 & 0 & 0 \\
a & 1 & 0 \\
b & \vartheta(a) & 1
\end{array}\right]: a, b \in F\right\}
$$

under matrix multiplication $U(a, b) U\left(a^{\prime}, b^{\prime}\right):=U\left(a+a^{\prime}, b+b^{\prime}+\right.$ $\left.a^{\prime} \vartheta(a)\right)$, where $F=G F\left(2^{n}\right)$ and $\vartheta$ be the Frobenius automorphism of $F$ given by $\vartheta(x)=x^{2}$ for all $x \in F$, then $\Gamma_{c}(G)=\left(2^{n}-1\right) K_{2^{n}}$.

(b) the Hanaki group $A(n, p)$ of order $p^{3 n}$ given by

$$
\left\{V(a, b, c)=\left[\begin{array}{lll}
1 & 0 & 0 \\
a & 1 & 0 \\
b & c & 1
\end{array}\right]: a, b, c \in F\right\}
$$

under matrix multiplication $V(a, b, c) V\left(a^{\prime}, b^{\prime}, c^{\prime}\right):=V\left(a+a^{\prime}, b+b^{\prime}+\right.$ $\left.c a^{\prime}, c+c^{\prime}\right)$, where $F=G F\left(p^{n}\right)$ and $p$ is a prime, then $\Gamma_{c}(G)=$ $\left(p^{n}+1\right) K_{p^{2 n}-p^{n}}$.

Now, we compute CN-spec $\left(\Gamma_{c}(G)\right)$ for more families of finite groups.

Theorem 12. Let $G$ be a non-abelian group.

(a) If $G$ is of order $p q$, where $p$ and $q$ are primes with $p \mid(q-1)$, then $\mathrm{CN}-\operatorname{spec}\left(\Gamma_{c}(G)\right)$ is given by

$$
\left\{(-(q-3))^{q-2},((q-2)(q-3))^{1},(-(p-3))^{p q-2 q},((p-2)(p-3))^{q}\right\} .
$$

(b) If $G$ is the quasidihedral group $Q D_{2^{n}}(n \geqslant 4)$, presented by $\langle a, b$ : $\left.a^{2^{n-1}}=b^{2}=1, b a b^{-1}=a^{2^{n-2}-1}\right\rangle$, then $\mathrm{CN}-\operatorname{spec}\left(\Gamma_{c}(G)\right)$ is given by

$$
\left\{\left(-\left(2^{n-1}-4\right)\right)^{2^{n-1}-3},\left(\left(2^{n-1}-3\right)\left(2^{n-1}-4\right)\right)^{1}, 0^{2^{n-1}}\right\} .
$$

(c) If $G$ is the projective special linear group $\operatorname{PSL}\left(2,2^{k}\right)$, where $k \geqslant 2$, then $\mathrm{CN}-\operatorname{spec}\left(\Gamma_{c}(G)\right)$ is given by

$$
\begin{aligned}
& \left\{\left(-\left(2^{k}-3\right)\right)^{\left(2^{k}+1\right)\left(2^{k}-2\right)},\left(\left(2^{k}-2\right)\left(2^{k}-3\right)\right)^{2^{k}+1},\right. \\
& \quad\left(-\left(2^{k}-4\right)\right)^{2^{k-1}\left(2^{k}+1\right)\left(2^{k}-3\right)},\left(\left(2^{k}-3\right)\left(2^{k}-4\right)\right)^{2^{k-1}\left(2^{k}+1\right)}, \\
& \left.\quad\left(-\left(2^{k}-2\right)\right)^{2^{k-1}\left(2^{k}-1\right)^{2}},\left(\left(2^{k}-1\right)\left(2^{k}-2\right)\right)^{2^{k-1}\left(2^{k}-1\right)}\right\} .
\end{aligned}
$$


(d) If $G$ is the general linear group $G L(2, q)$, where $q=p^{n}>2$ and $p$ is a prime, then $\mathrm{CN}-\operatorname{spec}\left(\Gamma_{c}(G)\right)$ is given by

$$
\begin{aligned}
& \left\{\left(-\left(q^{2}-3 q\right)\right)^{\frac{q(q+1)\left(q^{2}-3 q+1\right)}{2}},\left(\left(q^{2}-3 q+1\right)\left(q^{2}-3 q\right)\right)^{\frac{q(q+1)}{2}},\right. \\
& \quad\left(-\left(q^{2}-q-2\right)\right)^{\frac{q(q-1)\left(q^{2}-q-1\right)}{2}},\left(\left(q^{2}-q-1\right)\left(q^{2}-q-2\right)\right)^{\frac{q(q-1)}{2}}, \\
& \left.\quad\left(-\left(q^{2}-2 q-1\right)\right)^{(q+1)\left(q^{2}+2 q\right)},\left(\left(q^{2}-2 q\right)\left(q^{2}-2 q-1\right)\right)^{q+1}\right\} .
\end{aligned}
$$

Proof. (a) By Lemma 4(a), we have $\Gamma_{c}(G)=K_{q-1} \sqcup q K_{p-1}$. Therefore, by Theorem 1, we have

$\mathrm{CN}-\operatorname{spec}\left(\Gamma_{c}(G)\right)=$

$$
\left\{(-(q-3))^{q-2},((q-2)(q-3))^{1},(-(p-3))^{p q-2 q},((p-2)(p-3))^{q}\right\} .
$$

(b) By Lemma $4(\mathrm{~b})$, we have $\Gamma_{c}\left(Q D_{2^{n}}\right)=K_{2^{n-1}-2} \sqcup 2^{n-2} K_{2}$. Therefore, by Theorem 1, we have

$\mathrm{CN}-\operatorname{spec}\left(\Gamma_{c}\left(Q D_{2^{n}}\right)\right)=$

$$
\left\{\left(-\left(2^{n-1}-4\right)\right)^{2^{n-1}-3},\left(\left(2^{n-1}-3\right)\left(2^{n-1}-4\right)\right)^{1}, 0^{2^{n-1}}\right\} .
$$

(c) By Lemma 4(c), we have

$$
\Gamma_{c}(G)=\left(2^{k}+1\right) K_{2^{k}-1} \sqcup 2^{k-1}\left(2^{k}+1\right) K_{2^{k}-2} \sqcup 2^{k-1}\left(2^{k}-1\right) K_{2^{k}} .
$$

Therefore, by Theorem 1, we have

$$
\begin{aligned}
& \operatorname{CN}-\operatorname{spec}\left(\Gamma_{c}(G)\right)=\left\{\left(-\left(2^{k}-3\right)\right)^{\left(2^{k}+1\right)\left(2^{k}-2\right)},\left(\left(2^{k}-2\right)\left(2^{k}-3\right)\right)^{2^{k}+1},\right. \\
&\left(-\left(2^{k}-4\right)\right)^{2^{k-1}\left(2^{k}+1\right)\left(2^{k}-3\right)},\left(\left(2^{k}-3\right)\left(2^{k}-4\right)\right)^{2^{k-1}\left(2^{k}+1\right)}, \\
&\left.\left(-\left(2^{k}-2\right)\right)^{2^{k-1}\left(2^{k}-1\right)^{2}},\left(\left(2^{k}-1\right)\left(2^{k}-2\right)\right)^{2^{k-1}\left(2^{k}-1\right)}\right\} .
\end{aligned}
$$

(d) By Lemma 4(d), we have

$$
\Gamma_{c}(G)=\frac{q(q+1)}{2} K_{q^{2}-3 q+2} \sqcup \frac{q(q-1)}{2} K_{q^{2}-q} \sqcup(q+1) K_{q^{2}-2 q+1} .
$$

Therefore, by Theorem 1, we have

$$
\begin{aligned}
& \mathrm{CN}-\operatorname{spec}\left(\Gamma_{c}(G)\right)= \\
& \left\{\left(-\left(q^{2}-3 q\right)\right)^{\frac{q(q+1)\left(q^{2}-3 q+1\right)}{2}},\left(\left(q^{2}-3 q+1\right)\left(q^{2}-3 q\right)\right)^{\frac{q(q+1)}{2}},\right. \\
& \quad\left(-\left(q^{2}-q-2\right)\right)^{\frac{q(q-1)\left(q^{2}-q-1\right)}{2}},\left(\left(q^{2}-q-1\right)\left(q^{2}-q-2\right)\right)^{\frac{q(q-1)}{2}}, \\
& \left.\quad\left(-\left(q^{2}-2 q-1\right)\right)^{(q+1)\left(q^{2}+2 q\right)},\left(\left(q^{2}-2 q\right)\left(q^{2}-2 q-1\right)\right)^{q+1}\right\} .
\end{aligned}
$$

This completes the proof. 
Theorem 13. Let $G$ be a non-abelian group.

(a) If $G$ is the Hanaki group $A(n, \vartheta)(n \geqslant 2)$ of order $2^{2 n}$ given by

$$
\left\{U(a, b)=\left[\begin{array}{ccc}
1 & 0 & 0 \\
a & 1 & 0 \\
b & \vartheta(a) & 1
\end{array}\right]: a, b \in F\right\}
$$

under matrix multiplication $U(a, b) U\left(a^{\prime}, b^{\prime}\right):=U\left(a+a^{\prime}, b+b^{\prime}+\right.$ $\left.a^{\prime} \vartheta(a)\right)$, where $F=G F\left(2^{n}\right)$ and $\vartheta$ is the Frobenius automorphism of $F$ given by $\vartheta(x)=x^{2} \forall x \in F$, then $\mathrm{CN}-\operatorname{spec}\left(\Gamma_{c}(G)\right)$ is given by

$$
\left\{\left(-\left(2^{n}-2\right)\right)^{\left(2^{n}-1\right)^{2}},\left(\left(2^{n}-1\right)\left(2^{n}-2\right)\right)^{2^{n}-1}\right\} .
$$

(b) If $G$ is the Hanaki group $A(n, p)$ of order $p^{3 n}$ given by

$$
\left\{V(a, b, c)=\left[\begin{array}{lll}
1 & 0 & 0 \\
a & 1 & 0 \\
b & c & 1
\end{array}\right]: a, b, c \in F\right\}
$$

under matrix multiplication $V(a, b, c) V\left(a^{\prime}, b^{\prime}, c^{\prime}\right):=V\left(a+a^{\prime}, b+b^{\prime}+\right.$ $\left.c a^{\prime}, c+c^{\prime}\right)$, where $F=G F\left(p^{n}\right)$ and $p$ is a prime, then

$$
\begin{aligned}
& \mathrm{CN}-\operatorname{spec}\left(\Gamma_{c}(G)\right)=\left\{\left(-\left(p^{2 n}-p^{n}-2\right)\right)^{\left(p^{n}+1\right)\left(p^{2 n}-p^{n}-1\right)},\right. \\
&\left.\left(\left(p^{2 n}-p^{n}-1\right)\left(p^{2 n}-p^{n}-2\right)\right)^{p^{n}+1}\right\} .
\end{aligned}
$$

Proof. (a) By Lemma 5(a), we have $\Gamma_{c}(A(n, \vartheta))=\left(2^{n}-1\right) K_{2^{n}}$. Therefore, by Theorem 1 , we have

$$
\mathrm{CN}-\operatorname{spec}\left(\Gamma_{c}(A(n, \vartheta))\right)=\left\{\left(-\left(2^{n}-2\right)\right)^{\left(2^{n}-1\right)^{2}},\left(\left(2^{n}-1\right)\left(2^{n}-2\right)\right)^{2^{n}-1}\right\} \text {. }
$$

(b) By Lemma 5(b), we have $\Gamma_{c}(A(n, p))=\left(p^{n}+1\right) K_{p^{2 n}-p^{n}}$. Therefore, by Theorem 1, we have

$$
\begin{aligned}
& \mathrm{CN}-\operatorname{spec}\left(\Gamma_{c}(A(n, p))\right)=\left\{\left(-\left(p^{2 n}-p^{n}-2\right)\right)^{\left(p^{n}+1\right)\left(p^{2 n}-p^{n}-1\right)},\right. \\
&\left.\left(\left(p^{2 n}-p^{n}-1\right)\left(p^{2 n}-p^{n}-2\right)\right)^{p^{n}+1}\right\} .
\end{aligned}
$$

This completes the proof.

Note that all the groups considered above are abelian centralizer group (in short, AC-group). In other words, $C_{G}(x)$ is abelian for all $x \in G \backslash Z(G)$. In the following two results we compute CN-spectrum of commuting graphs of finite AC-groups. 
Theorem 14. Let $G$ be a finite non-abelian $A C$-group with distinct centralizers $X_{1}, \ldots, X_{n}$ of non-central elements of $G$. Then $\mathrm{CN}-\operatorname{spec}\left(\Gamma_{c}(G)\right)$ is given by the set

$$
\begin{aligned}
& \left\{\left(-\left(\left|X_{1}\right|-|Z(G)|-2\right)\right)^{\left|X_{1}\right|-|Z(G)|-1}\right. \\
& \left(\left(\left|X_{1}\right|-|Z(G)|-1\right)\left(\left|X_{1}\right|-|Z(G)|-2\right)\right)^{1}, \ldots \\
& \left(-\left(\left|X_{n}\right|-|Z(G)|-2\right)\right)^{\left|X_{n}\right|-|Z(G)|-1} \\
& \left.\quad\left(\left(\left|X_{n}\right|-|Z(G)|-1\right)\left(\left|X_{n}\right|-|Z(G)|-2\right)\right)^{1}\right\} .
\end{aligned}
$$

Proof. By [12, Lemma 1], we have $\Gamma_{c}(G)=\stackrel{\sqcup}{i=1}^{n} K_{\left|X_{i}\right|-|Z(G)|}$. Therefore, the result follows from Theorem 1.

Corollary 3. Let $G \cong H \times A$ where $H$ is a finite non-abelian $A C$-group and $A$ is any finite abelian group. Then $\mathrm{CN}-\operatorname{spec}\left(\Gamma_{c}(H \times A)\right)$ is given by the set

$$
\begin{aligned}
& \left\{\left(-\left(\left(\left|X_{1}\right|-|Z(H)|\right)|A|-2\right)\right)^{\left(\left|X_{1}\right|-|Z(H)|\right)|A|-1}\right. \\
& \left(\left(\left(\left|X_{1}\right|-|Z(H)|\right)|A|-1\right)\left(\left(\left|X_{1}\right|-|Z(H)|\right)|A|-2\right)\right)^{1}, \ldots \\
& \left(-\left(\left(\left|X_{n}\right|-|Z(H)|\right)|A|-2\right)\right)^{\left(\left|X_{n}\right|-|Z(H)|\right)|A|-1} \\
& \left.\quad\left(\left(\left(\left|X_{n}\right|-|Z(H)|\right)|A|-1\right)\left(\left(\left|X_{n}\right|-|Z(H)|\right)|A|-2\right)\right)^{1}\right\}
\end{aligned}
$$

where $X_{1}, \ldots, X_{n}$ are the distinct centralizers of non-central elements of $H$.

Proof. Let $H$ be a finite non-abelian AC-group and $A$ be any finite abelian group then $Z(H \times A)=Z(H) \times A$. Further, if $X_{1}, \ldots, X_{n}$ are the distinct centralizers of non-central elements of $H$ then the distinct centralizers of non-central elements of $H \times A$ are given by $X_{1} \times A, X_{2} \times A, \ldots, X_{n} \times A$. Therefore, $H \times A$ is also an AC-group. Hence, the result follows from Theorem 14.

\section{Consequences}

In this section, we record some consequences of the results obtained in earlier sections. Firstly, note that CN-spectrum of commuting graphs of all the groups considered in section 3 and section 4 contain only integers. Therefore, commuting graphs of those groups are CN-integral. The following results show that the commuting graph of a finite $n$-centralizer group is CN-integral if $n=4,5$. 
Theorem 15. If $G$ is a finite 4-centralizer group then $\Gamma_{c}(G)$ is $C N$ integral.

Proof. Let $G$ be a finite 4-centralizer group. Then, by Theorem 2, we have $\frac{G}{Z(G)} \cong \mathbb{Z}_{2} \times \mathbb{Z}_{2}$. Hence the result follows from Theorem 11(b) by considering $p=2$.

Further, we have the following result.

Theorem 16. Let $G$ be a finite $(p+2)$-centralizer $p$-group for any prime $p$. Then $\Gamma_{c}(G)$ is $C N$-integral.

Proof. Let $G$ be a finite $(p+2)$-centralizer $p$-group. Then, by Theorem 3, we have $\frac{G}{Z(G)} \cong \mathbb{Z}_{p} \times \mathbb{Z}_{p}$. Hence the result follows from Theorem 11(b).

Theorem 17. If $G$ is a finite 5-centralizer group then $\Gamma_{c}(G)$ is $C N$ integral.

Proof. Let $G$ be a finite 5 -centralizer group. Then by Theorem 4 we have $\frac{G}{Z(G)} \cong \mathbb{Z}_{3} \times \mathbb{Z}_{3}$ or $D_{6}$. Hence the result follows from Theorem 11, parts (b) and (c).

As a corollary to Theorem 15 and Theorem 17 we have the following result.

Corollary 4. Let $G$ be a finite non-abelian group and $\left\{x_{1}, x_{2}, \ldots, x_{r}\right\}$ be a set of pairwise non-commuting elements of $G$ having maximal size. Then $\Gamma_{c}(G)$ is CN-integral if $r=3,4$.

Proof. By Theorem 5, we have that $G$ is a 4-centralizer or a 5-centralizer group. Hence the result follows from Theorem 15 and Theorem 17.

The following theorems give some rational numbers $r$ such that $\Gamma_{c}(G)$ is CN-integral if $\operatorname{Pr}(G)=r$, where $\operatorname{Pr}(G)$ is the commutativity degree of a finite group $G$.

Theorem 18. If $\operatorname{Pr}(G) \in\left\{\frac{5}{14}, \frac{2}{5}, \frac{11}{27}, \frac{1}{2}, \frac{7}{16}, \frac{5}{8}\right\}$ then $\Gamma_{c}(G)$ is CN-integral. Proof. If $\operatorname{Pr}(G) \in\left\{\frac{5}{14}, \frac{2}{5}, \frac{11}{27}, \frac{1}{2}, \frac{7}{16}, \frac{5}{8}\right\}$ then as shown in [24, pp. 246] and [20, pp. 451], we have $\frac{G}{Z(G)}$ is isomorphic to one of the groups in $\left\{D_{14}, D_{10}, D_{8}, D_{6}, \mathbb{Z}_{2} \times \mathbb{Z}_{2}, \mathbb{Z}_{3} \times \mathbb{Z}_{3}\right\}$. Hence the result follows from Theorem 11, parts (b) and (c).

Theorem 19. Let $G$ be a finite group and $p$ the smallest prime divisor of $|G|$. If $\operatorname{Pr}(G)=\frac{p^{2}+p-1}{p^{3}}$ then $\Gamma_{c}(G)$ is CN-integral. 
Proof. If $\operatorname{Pr}(G)=\frac{p^{2}+p-1}{p^{3}}$ then, by Theorem 6, we have $\frac{G}{Z(G)}$ is isomorphic to $\mathbb{Z}_{p} \times \mathbb{Z}_{p}$. Hence the result follows from Theorem $11(\mathrm{~b})$.

Theorem 20. If $G$ is a finite non-solvable group with $\operatorname{Pr}(G)=\frac{1}{12}$ then $\Gamma_{c}(G)$ is $C N$-integral.

Proof. By Theorem 7, we have that $G$ is isomorphic to $A_{5} \times B$ for some finite abelian group $B$. Since $A_{5}$ is an AC-group, the result follows from Corollary 3.

The following three theorems show that $\Gamma_{c}(G)$ is CN-integral if $\Gamma_{c}(G)$ is planar and $G$ is not isomorphic to $S_{4}$, troidal or the complement of $\Gamma_{c}(G)$ is planar.

Theorem 21. Let $G$ be a finite non-abelian group. If $\Gamma_{c}(G)$ is planar and $G$ is not isomorphic to $S_{4}$ then $\Gamma_{c}(G)$ is $C N$-integral.

Proof. By Theorem 8, $G$ is isomorphic to either $D_{6}, D_{8}, D_{10}, D_{12}, Q_{8}$, $Q_{12}, \mathbb{Z}_{2} \times D_{8}, \mathbb{Z}_{2} \times Q_{8}, \mathcal{M}_{16}, \mathbb{Z}_{4} \rtimes \mathbb{Z}_{4}, D_{8} * \mathbb{Z}_{4}, S G(16,3), A_{4}, A_{5}, S_{4}$, $S L(2,3)$ or $S z(2)$.

If $G \cong D_{6}, D_{8}, D_{10}, D_{12}, Q_{8}$ or $Q_{12}$ then, by Corollary 2 parts (c) and (d), we have that $\Gamma_{c}(G)$ is CN-integral. If $G \cong \mathbb{Z}_{2} \times D_{8}, \mathbb{Z}_{2} \times Q_{8}, \mathcal{M}_{16}, \mathbb{Z}_{4} \rtimes$ $\mathbb{Z}_{4}, D_{8} * \mathbb{Z}_{4}$ or $S G(16,3)$ then, by Corollary 1 , it follows that $\Gamma_{c}(G)$ is $\mathrm{CN}$-integral. If $G \cong A_{4}$ then it can be seen that $\Gamma_{c}(G)=K_{3} \sqcup 4 K_{2}$. Using Theorem 1, we have CN-spec $\left(\Gamma_{c}(G)\right)=\left\{(-1)^{2}, 2^{1}, 0^{8}\right\}$, hence $\Gamma_{c}(G)$ in CNintegral. If $G \cong S z(2)$ then $\frac{G}{Z(G)} \cong S z(2)$. Therefore, by Theorem 11(a), it follows that $\Gamma_{c}(G)$ is CN-integral. If $G$ is isomorphic to $S L(2,3)$ then it can be seen that $\Gamma_{c}(G)=3 K_{2} \sqcup 4 K_{4}$. Therefore, by Theorem 1, we have CN-spec $\left(\Gamma_{c}(G)\right)=\left\{0^{6},(-2)^{12}, 6^{4}\right\}$, hence $\Gamma_{c}(G)$ in CN-integral.

We have $P S L(2,4) \cong A_{5}$. Therefore, if $G \cong A_{5}$ then by Theorem $12(\mathrm{c})$ it follows that $\Gamma_{c}(G)$ is $\mathrm{CN}$-integral.

Finally, if $G \cong S_{4}$ then it can be seen that the characteristic polynomial of $C N\left(\Gamma_{c}(G)\right)$ is $x^{8}(x-3)^{2}(x+1)^{11}\left(x^{2}-5 x-30\right)$ and so

$$
\mathrm{CN}-\operatorname{spec}\left(\Gamma_{c}(G)\right)=\left\{0^{8}, 3^{2},(-1)^{11},\left(\frac{5+\sqrt{145}}{2}\right)^{1},\left(\frac{5-\sqrt{145}}{2}\right)^{1}\right\} .
$$

Hence, $\Gamma_{c}(G)$ is not CN-integral. This completes the proof.

Theorem 22. Let $G$ be a finite non-abelian group. If $\Gamma_{c}(G)$ is toroidal then $\Gamma_{c}(G)$ is $C N$-integral. 
Proof. By Theorem 9, $G$ is isomorphic to either $D_{14}, D_{16}, Q_{16}, Q D_{16}$, $D_{6} \times \mathbb{Z}_{3}, A_{4} \times \mathbb{Z}_{2}$ or $\mathbb{Z}_{7} \rtimes \mathbb{Z}_{3}$.

If $G \cong D_{14}, D_{16}$ or $Q_{16}$ then, by Corollary 2 parts (c) and (d), it follows that $\Gamma_{c}(G)$ is CN-integral. If $G \cong Q D_{16}$ then, by Theorem $12(\mathrm{~b})$, we have that $\Gamma_{c}(G)$ is CN-integral. If $G \cong \mathbb{Z}_{7} \rtimes \mathbb{Z}_{3}$ then $\Gamma_{c}(G)$ is CN-integral, follows from Theorem 12 (a) by considering $p=3$ and $q=7$. If $G$ is isomorphic to $D_{6} \times \mathbb{Z}_{3}$ then $\frac{G}{Z(G)} \cong D_{6}$. Therefore, by Theorem 11(c), $\Gamma_{c}(G)$ is CN-integral. If $G$ is isomorphic to $A_{4} \times \mathbb{Z}_{2}$ then by Corollary 3 it follows that $\Gamma_{c}(G)$ is CN-integral since $A_{4}$ is an AC-group. This completes the proof.

We also have the following result.

Theorem 23. Let $G$ be a finite non-abelian group. If the complement of $\Gamma_{c}(G)$ is planar then $\Gamma_{c}(G)$ is CN-integral.

Proof. By Theorem 10, $G$ is isomorphic to either $D_{6}, D_{8}$ or $Q_{8}$. Hence the result follows from Corollary 2 parts (c) and (d).

In $[12,13,21]$, Dutta and Nath have computed spectrum of the commuting graphs of all the groups considered in this paper. It was observed that commuting graphs of all those groups except $S_{4}$ are integral. The commuting graph of $S_{4}$ is neither integral nor CN-integral. Recall that a graph is called integral if all the eigenvalues of its adjacency matrix are integers. We conclude this paper with the following problems.

Problem 1. Let $G$ be a finite non-abelian group. Does the fact " $\Gamma_{c}(G)$ is integral" imply $\Gamma_{c}(G)$ is CN-integral?

More generally, one may pose the following problem.

Problem 2. Let $\mathcal{G}$ be any graph. Does the fact " $\mathcal{G}$ is integral" imply $\mathcal{G}$ is CN-integral?

\section{Acknowledgment}

The authors would like to thank the referee for his/her valuable comments and suggestions. The first author is thankful to Indian Council for Cultural Relations for the ICCR Scholarship. 


\section{References}

[1] A. Abdollahi, S. Akbari and H. R. Maimani, Non-commuting graph of a group, J. Algebra, 298, 468-492 (2006).

[2] A. Abdollahi, S. M. Jafarain and A. M. Hassanabadi, Groups with specific number of centralizers, Houston J. Math., 33(1), 43-57 (2007).

[3] M. Afkhami, M. Farrokhi D. G. and K. Khashyarmanesh, Planar, toroidal, and projective commuting and non-commuting graphs, Comm. Algebra, 43(7), 29642970 (2015).

[4] A. Alwardi, N. D. Soner and I. Gutman, On the common-neighborhood energy of a graph, Bulletin T.CXLIII de l'Académie serbe des sciences et des arts, No. 36, 49-59 (2011).

[5] S. Akbari, A. Mohammadian, H. Radjavi and P. Raja, On the diameters of commuting graphs, Linear Algebra Appl., 418, 161-176 (2006).

[6] A. R. Ashrafi, On finite groups with a given number of centralizers, Algebra Colloq., 7(2), 139-146 (2000).

[7] S. M. Belcastro and G. J. Sherman, Counting centralizers in finite groups, Math. Magazine, 67(5), 366-374 (1994).

[8] A. Castelaz, Commutativity degree of finite groups, M.A. thesis, Wake Forest University (2010).

[9] A. K. Das, R. K. Nath and M. R. Pournaki, A survey on the estimation of commutativity in finite groups, Southeast Asian Bull. Math., 37(2), 161-180 (2013).

[10] A. K. Das and D. Nongsiang, On the genus of the commuting graphs of finite non-abelian groups, Int. Electron. J. Algebra, 19, 91-109 (2016).

[11] J. Dutta, A study of finite groups in terms of their centralizers, M. Phil. thesis, North-Eastern Hill University (2010).

[12] J. Dutta and R. K. Nath, Spectrum of commuting graphs of some classes of finite groups, Matematika, 33(1), 87-95 (2017).

[13] J. Dutta and R. K. Nath, Finite groups whose commuting graphs are integral, Mat. Vesnik, 69(3), 226-230 (2017).

[14] J. Dutta and R. K. Nath, Laplacian and signless Laplacian spectrum of commuting graphs of finite groups, Khayyam J. Math., 4(1), 77-87 (2018).

[15] P. Erdös and P. Turán, On some problems of a statistical group-theory IV, Acta. Math. Acad. Sci. Hungar., 19, 413-435 (1968).

[16] A. Iranmanesh and A. Jafarzadeh, Characterization of finite groups by their commuting graph, Acta Mathematica Academiae Paedagogicae Nyiregyhaziensis, 23(1), 7-13 (2007).

[17] D. MacHale, How commutative can a non-commutative group be?, Math. Gaz., 58, 199-202 (1974).

[18] G. L. Morgan and C. W. Parker, The diameter of the commuting graph of a finite group with trivial center, J. Algebra, 393(1), 41-59 (2013).

[19] R. K. Nath, Commutativity degrees of finite groups - a survey, M. Phil. thesis, North-Eastern Hill University (2008). 
[20] R. K. Nath, Commutativity degree of a class of finite groups and consequences, Bull. Aust. Math. Soc., 88(3), 448-452 (2013).

[21] R. K. Nath, Various specta of commuting graphs n-centralizer finite groups, Int. J. Eng. Science Tech., 10(2S), 170-172 (2018).

[22] R. K. Nath and A. K. Das, On a lower bound of commutativity degree, Rend. Circ. Math. Palermo, 59(1), 137-141 (2010).

[23] C. Parker, The commuting graph of a soluble group, Bull. London Math. Soc., 45(4), 839-848 (2013).

[24] D. J. Rusin, What is the probability that two elements of a finite group commute?, Pacific J. Math., 82(1), 237-247 (1979).

\section{Walaa Nabil \\ Taha Fasfous, Rajat Kanti Nath}

Reza Sharafdini

\section{CONTACT INFORMATION}

Department of Mathematical Sciences, Tezpur University, Napaam-784028, Sonitpur, Assam, India

$$
\begin{aligned}
& E-\operatorname{Mail}(s): \text { w.n.fasfous@gmail.com, } \\
& \text { rajatkantinath@yahoo.com }
\end{aligned}
$$

Web-page(s): www.tezu.ernet.in/dmaths/ people/faculty-pages/rkn. html

Department of Mathematics, Faculty of Science, Persian Gulf University, Bushehr 75169-13817, Iran

E-Mail(s): sharafdini@pgu.ac.ir

Web-page(s): research.pgu.ac.ir/

$\sim$ RSharafdini

Received by the editors: 09.02.2019. 